\title{
Saskatchewan Christmas Bird Count, 1959
}

\author{
Edited by Mary Houston, Yorkton.
}

The best single observation this year was that of Steve Waycheshen, who noted a Barred Owl during his regular count, and then was able to capture the bird so that it could be banded on January 2. Other good owl records were of a Boreal Owl at Prince Albert and a Hawk Owl at Big River. Snowy Owls were unusually scarce and noted only at Dodsland and Melville during the count itself, though seen at Battleford and Bladworth on other days during the Christmas season. Goshawks were also scarce, being seen only at High Hill. Golden Eagles, however, were reported from seven localities and the Pigeon Hawk from four.

In addition to the Barred Owl (incidentally only the fifth record for Saskatchewan), new species not listed during seventeen previous Christmas seasons included a Cooper's Hawk noted by Glen A. Fox and Spencer Sealy at Battleford on Dec. 28, and a Peregrine Falcon noted at Moose Jaw on Dec. 28 and at Regina on Dec. 26. This brings the total of species noted during 18 Christmas seasons to a remarkable 97 species.

Other records of interest include a Sparrow Hawk at Skull Creek, Flickers at Craven, Regina and Moose Jaw, a Robin at Craven and Regina, Cedar Waxwings at Estevan, Regina and Fort San, a Meadowlark at Fort San and Regina, a Whitethroated Sparrow at Madge Lake, and a Song Sparrow at Spirit Lake. Blackbirds were represented by Brewer's at Saskatoon, Rusties at Craven and Fort San, and Redwings at Craven, Fort San and Skull Creek.

BANGOR, Sask. Dec. 26; about farm and 9 miles by car; temp. $20^{\circ}$; calm, cloudy. 10 species, 234 individuals. Ruffed Grouse, 1; Sharp-tailed Grouse, 5; Hairy Woodpecker, 1; Downy Woodpecker, 2; Black-billed Magpie, 6; Black-capped Chickadee, 4; Hcuse Sparrow, 50; Pine Grosbeak, 9; Common Redpoll, 48; Sriow Bunting, 108.-Mrs. A. Thompson.

BATTLEFORD, Sask. Dec. $26 ; 8$ miles on foot and 23 miles by car in $81 / 4$ hours; temp. $21^{\circ}$; wind N.W. 15 mph.; cloudy, snowing; 6 inches snow on ground. 19 species, 2032 individuals. Golden Eagle, 2; Ruffed Grouse, 2; Sharp-tailed Grouse, 32; Ring-necked Pheasant, 6; Gray Partridge, 8; Rock Dove, 267; Great Horned Owl, 1; Hairy Woodpecker, 2; Downy Woodpecker, 4; Blue Jay, 2; Black-billed Magpie, 2; Black-capped Chickadee, 15; Bohemian Waxwing, 273; Starling, 1; House Sparrow, 354; Pine Grosbeak, 24; Common Redipoll, 637; Slate-colored Junco, 2; Snow Bunting, 380. (Add: Cooper's Hawk, 1, Dec. 28; Pigeon Hawk, 1, Dec. 27; Snowy Owl, 1, Dec. 24) -Glen A. Fox, Spencer Sealy (compiler).

BIG RIVER, Sask. Dec. 30; 1 mile on foot in $1 \frac{1 / 2}{2}$ hours and 24 miles by truck in $11 / 2$ hours; temp. $20^{\circ}$, calm, cloudy. Total, 13 species, 149 individuals. Great Horned Owl, 2; Hawk Owl, 1; Pileated Woodpecker, 2; Hairy Woodpecker, 10; Downy Woodpecker, 6; Blue Jay, 5; Black-billed Magpie, 3; Common Raven, 5; Blackcapped Chickadee, 26; Nuthatch (sp.?), 2; Pine Grosbeak, 7; Common Redpoll, 30; Snow Bunting, 50. (Add: Ruffed Grouse, 2; Sharp-tailed Grouse, 8) -Mrs. Anne Olson.

BLADWORTH, Sask. Dec. $28 ; 4 \frac{1}{2}$ hours on foot; temp. $16^{\circ}$ to $20^{\circ}$; calm, foggy and overcast; 12 inches snow. 8 species, 368 individuals. Sharp-tailed Grouse, 20; Gray Partridge, 41; Great Horned Owl, 2; Downy Woodpecker, 1; Black-billed Magpie, 6; House Sparrow, 27; Common Redpoll, 246; Snow Bunting, 25. (Add: Rock Dove, 17, Dec. 31; Snowy Owl, 1, Dec. 24; Starling, 2, Dec. 31) -Kenneth, Margaret, Sam and $P$. Lawrence Beckie.

CRAVEN, Sask. Dec. 28; 42 party miles on foot and 144 party miles by car in $7 \frac{1}{2}$ hours ( 7 observers in 3 parties); temp. $23^{\circ}$ to $25^{\circ}$; calm. 17 species; 672 individuals. Sharp-tailed Grouse, 6; Gray Partridge, 7; Great Horned Owl, 3; Yellow-shafted Flicker, 1; Hairy Woodpecker, 10; Downy Woodpecker, 10; Blue Jay, 1; Black-billed Magpie, 62; Black- 
capped Chickadee, 50; Robin, 2; Starling, 1; House Sparrow, 342; Redwinged Blackbird, 2; Rusty Blackbird, 3; Pine Grosbeak, 5; Common Redpoll, 160; Snow Bunting, 7.-F. G. Bard, F. Brazier (compiler), E. Fox, R. Fox, B. McCorquodale, R. W. Nero, A. Swanston.

DILKE, Sask. Dec. $25 ; 2 \frac{1}{2}$ miles on foot, by team and horseback, 3 hours by car; temp $25^{\circ}$ to $31^{\circ}$; wind 20 to $35 \mathrm{mph}$; overcast; 5 inches snow. 8 species, 275 individuals. Gray Partridge, 50; Great Horned Owl, 1; Horned Lark, 3; Blue Jay, 1; Blackbilled Magpie, 7; House Sparrow, 190; Common Redpoll, 4; Snow Bunting, 20. (Add: Sharp-tailed Grouse, Dec. 28 and Jan 3; Starling, Dec. 26, 31, and Jan. 3).-J. B. Belcher (compiler), Mr. and Mrs. S. R. Belcher, Margaret Belcher.

DODSLAND, Sask. Dec. 23; 2 mi. on foot and 64 by car in $91 / 2$ hours; temp. $5^{\circ}$; wind NW 0 to $10 \mathrm{mph}$; ; clear. 13 species, 330 individuals. Golden. Eagle, 1; Pigeon Hawk, 1; Sharptailed Grouse, 95; Gray Partridge, 19; Rock Dove, 2; Snowy Owl, 2; Shorteared Owl, 2; Horned Lark, 2; Blackbilled Magpie, 8; House Sparrow, 4; Hoary Redpoll, 2; Common Redpoll, 42; Snow Bunting, 150.-Richard Fyfe.

DUNDURN, Sask Dec. 25; 2 miles on foot; temp. $18^{\circ}$ to $20^{\circ}$; wind light. 3 species, 202 individuals. Blackbilled Magpie, 2; Starling, 100; House Sparrow, 100. (Add. Sharp-tailed Grouse, Dec. 23; Common Redpoll, Dec. 24, 27, 29, 31, and Jan. 1).Edgar W. Sullivan

ESTEVAN, Sask. Dec. 28; 5 miles on foot in 6 hours; temp. $22^{\circ} ; 18$ inches of snow. 8 species, 205 individuals. Sharp-tailed Grouse, 7; Rock Dove, 14; Downy Woodpecker, 3; Blackbilled Magpie, 7; Black-capped Chickadee, 9; Bohemian Waxwing, 11; House Sparrow, 132; Snow Bunting, 22. (Add. Mallard, 2, Dec. 30; Gray Partridge, 7, Dec. 31; Cedar Waxwing, 3, Dec. 30; Starling, 12, Dec. 26).-Darrel Carlson, Ross Lein (compiler).

FORT SAN, Sask. Dec. 25; 7 miles by car and 1 mile on foot in $2 \frac{1}{2}$ hours; temp. $27^{\circ}$; wind S.E.; cloudy; 5 inches snow. 9 species, 125 individuals. Ruffed Grouse, 1; Downy
Woodpecker, 1; Black-billed Magpie, 11; Black-capped Chickadee, 3; Cedar Waxwing, 25; House Sparrow, 105; Redwinged Blackbird, 1; Rusty Blackbird, 3; Common Redpoll, 2. (Add: Sharp-tailed Grouse, 2, Dec. 27; Gray Partridge, 8, Dec. 28; Hairy Woodpecker, 1, Dec. 26; Bohemian Waxwing, 2, Dec. 26; Western Meadowlark, 1, Dec. 23; Pine Grosbeak, 1, Dec. 28).-Dr. G. D. Barnett, E. M. Callin (compiler), Errol Cochrane, Dr. H. D. Jenner, Jack Lowe, S. P. Regan.

KAMSACK, Sask. Dec. 29; 4 miles by foot; 8 hours; temp. $20^{\circ}$ to $30^{\circ}$; no wind; overcast. 11 species, 264 individuals. Ruffed Grouse, 1; Sharptailed Grouse, 28; Great Horned Owl, 1; Downy Woodpecker, 1; Blackbilled Magpie, 10; Black-capped Chickadee, 28; Bohemian Waxwing, 45; Starling, 2; Evening Grosbeak, 10; Pine Grosbeak, 110; Common Redpoll, 28.-Lawrence Ostoforoff.

KEATLEY, Sask. Dec. 24; 25 miles by truck and 2 miles on foot. 7 species, 2308 individuals. Sharptailed Grouse, 7; Black-billed Magpie, 9; Black-capped Chickadee, 5; Bohemian Waxwing, 7; House Sparrow, 30; Common Redpoll, 2,000; Snow Bunting, 250. (Add. Golden Eagle, 1; Ruffed Grouse, 1).-A. P. Pym.

KELVINGTON - HIGH HILL, Sask. Jan. 2, 1960; 4 hours by car and on foot; temp. $10^{\circ}$; strong wind; overcast. 10 species, 558 individuals. Goshawk, 1; Golden Eagle, 1; Ruffed Grouse, 1; Gray Jay, 1; Black-billed Magpie, 4; Black-capped Chickadee, 4; House Sparrow, 156; Pine Grosbeak, 60; Common Redpoll, 189; Snow Bunting, 150.-Gary Anweiler, Dr. and Mrs. Stuart Houston (compilers), Anton and Steve Waycheshen.

KINDERSLEY, Sask. Jan. 3， 1960; 5 miles by foot in 3 hours and 11 miles by car in 2 hours; temp. $-10^{\circ}$; wind NW at 10 ; clear. 9 species, 786 individuals. Sharp-tailed Grouse, 1; Gray Partridge, 57; Rock Dove, 200; Black-billed Magpie, 3; Bohemian Waxwing, 8; Starling, 5; House Sparrow, 410; Common Redpoll, 2; Snow Bunting, 100.-Glen A. Fox.

KLOGEI LAKE-HIGH HILL, Sask. Dec. $27 ; 16$ miles on foot in 7 hours; $10^{\circ}$ to $20^{\circ}$; wind north; cloudy with 
intermittent snow; light and heavy bush and small lakes. 13 species, 28 individuals. Ruffed Grouse, 2; Great Horned Owl, 1; Barred Owl, 1; Hairy Woodpecker, 1; Gray Jay, 2; Blue Jay, 1; Black-billed Magpie, 2; Common Raven, 3; Black-capped Chickadee, 5; Boreal Chickadee, 2; Evening Grosbeak, 2; Pine Grosbeak, 1; Common Redpoll, 5. (Add: Pileated Woodpecker, 1, Dec. 30; Bohemian Waxwing, 36, Dec. 23).-Steve Waycheshen.

LEADER, Sask. Jan. 3, 1960. 3 species, 34 individuals. Sharp-tailed Grouse, 8; Ring-necked Pheasant, 16; Black-billed Magpie, 10. (Add: Snow Bunting, 300, Dec. 31).-Daisy Meyers.

MADGE LAKE, Sask. (Center on southwest corner of Duck Mountain Provincial Park). Jan. 1, 1960; 46 miles and 8 hours by car, $21 / 2$ miles and 2 hours on foot; temp. $10^{\circ}$ to $15^{\circ}$; wind north at 5 to $15 \mathrm{mph}$; 15 inches of snow. 17 species, 410 individuals. Golden Eagle, 1; Ruffed Grouse, 12; Hairy Woodpecker, 1; Downy Woodpecker, 3; Northern Three-toed Woodpecker, 1; Gray Jay, 6; Blue Jay, 11; Black-billed Magpie, 15; Common Raven, 1; Black-capped Chickadee, 20; Bohemian Waxwing, 86; House Sparrow, 47; Evening Grosbeak, 7; Pine Grosbeak, 12; Common Redpoll, 74; White-throated Sparrow, 1; Snow Bunting, 112.-Gary Anweiler, Bill Horseman, Dr. and Mrs. Stuart Houston (compilers), Jacob Jmaeff.

MASEFIELD, Sask. Dec. 29; 3 miles on foot and 48 by car in 6 hours; temp. $28^{\circ}$; wind NNW light; sunny; 6 inches of snow. 9 species, 243 individuals. Golden Eagle, 2; Sharptailed Grouse, 3; Ring-necked Pheasant, 21; Horned Lark, 22; Black-billed Magpie, 11; Black-capped Chickadee, 4; House Sparrow, 173; Common Redpoll, 6; Snow Bunting, 1. (Add: Gray Partridge, 5, Dec. 24; Starling, 9, Dec. 28; Cowbird (Rusty Blackubird? -Ed.), 11, Jan 2).-J. David Chandler.

MEATH PARK, Sask. Dec. $25 ; 33 \frac{3}{4}$ miles on foot in $1 \frac{1}{2}$ hours and 5 miles by car in $11 / 2$ hours; temp. $18^{\circ}$; wind NNE 8 mph.; overcast; 10-14 inches of snow. 9 species, 82 individuals. Ruffed Grouse, 2; Sharptailed Grouse, 4; Blue Jay, 2; Black- billed Miagpie, 1; Black-capped Chickadee, 4; Bohemian Waxwing, 1; House Sparrow, 14; Pine Grosbeak, 1; Common Redpoll, 53.-Jim Hrenyk and Pete Hrenyk.

MELVILLE, Sask. Dec. 28; 8 miles on foot in 5 hours; temp. $10^{\circ}$; wind light; 16 inches of snow. 6 species, 263 individuals. Snowy Owl, 3; Shorteared Owl, 1; Downy Woodpecker, 1; Black-billed Magpie, 5; House Sparrow, 250; Common Redpoll, 3. (Add: Ruffed Grouse, 1; Black-capped Chickadee, 3).-Gary Anweiler.

MOOSE JAW, Sask. Dec. 26; 6 miles and 4 hours on foot (14 observers in two parties); temp. 24; wind NW at 5 to $10 \mathrm{mph}$; overcast with light snow. 1.1 species, 249 individuals. Sharptailed Grouse, 12; Ring-necked Pheasant, 4; Gray Partridge, 4; Rock Dove, 75; Yellow-shafted Flicker, 2; Downy Woodpecker, 1; Black-billed Magpie, 35; Black-capped Chickadee, 10; Golden-crowned Kinglet, 2; House Sparrow, 100; Common Redpoll, 4. (Add on Dec. 28: Peregrine Falcon, 1; Barred Owl (?) 1; Bohemian Waxwing, 12; Starling, 24, Pine Grosbeak, 4.) Carl Ellis (compiler), John Ellis, Dr. and Mrs. D. M. Ewart, Patty Ewart, Douglas Ewart, Neil Farrell, John Horton, Patrick Kennedy, Mrs. A. J. Rankin, Miss Molly Ritchie, Mrs. D. Rhodes, Mrs. Remington Walker (Moose Jaw Natural History Society.)

NIPAWIN, Sask. Dec. 26; about town; mild; snow falling; one foot of snow on ground. 12 species, 175 individuals. Ruffed Grouse, 1; Hairy Woodpecker, 2; Downy Woodpecker, 1; Gray Jay, 1; Blue Jay, 2; Black-billed Magpie, 1; Common Raven, 5; Black-capped Chickadee, 11; House Sparrow, 100; Evening Grosbeak, 6; Pine Grosbeak, 23; Common Redpoll, 22.- Maurice G. Street.

PRINCE ALBERT, Sask. Dec. 27; 6 miles by car in $2 \frac{1}{2}$ hours; temp. $15^{\circ}$ to $25^{\circ} ; 12$ inches of snow. 8 species and 29 individuals. Rock Dove, 2; Boreal Owl, 1; Black-billed Magpie, 1; Boreal Chickadee, 6; House Sparrow, 2; Evening Grosbeak, 2; Pine Grosbeak, 6; Common Redpoll, 9. (Add on Dec. 28: Gray Jay, 1; Blackcapped Chickadee, 6 ; Bohemian Waxwing, 30).-E. W. Brooman, A. Capusten, E. Evasiuk, D. Karasiuk (compiler.) 
REGINA, Sask. Dec. $26 ; 44$ miles on foot and 135 by car; 32 party hours on foot, 45 by car; temp. $26^{\circ}$, wind NE at $10 ; 4-10$ inches snow; 23 species, 2112 individuals. Horned Grebe, 1; White Pelican, 2; Whistling Swan, 3; Mute Swan, 4; Canada Goose, 185; Mallard, 300; Pintail, 1; Prairie Falcon, 1; Peregrine Falcon, 1; Gray Partridge, 8; American Coot, 1; Yellow-shafted Flicker, 2; Downy Woodpecker, 3; Black-billed Magpie, 27; Black-capped Chickadee, 16; Robin, 3; Bohemian Waxwing, 26; Cedar Waxwing, 3; Northern Shrike, 1; House Sparrow, $1520+$; Western Meadowlark, 1; Common Grackle, 1; Evening Grosbeak, 1.-F. Brazier, E. Cruickshank, H. Erikson, E. L. Fox (compiler), R. Fox, R. Knutson, G. F. Ledingham, R. McCall, J. Moore, R. W. Nero, C. Willway.

SASKATOON, Sask. Dec. 26 ; 9 party miles on foot in 10 hours, 114 party miles by car in 113/4 hours; 23 observers in six parties; temp. $20^{\circ}$; wind $\mathrm{NW}$ at $10 \mathrm{mph}$; overcast; snowing; 8 inches of snow on ground. 16 species, 1,581 individuals. Mallard, 9; Pigeon Hawk, 1; Gray Partridge, 23; Rock Dove, 22; Blue Jay, 2; Blackbilled Magpie, 35; Black-capped Chickadee, 17; Bohemian Waxwing, 148; Northern Shrike, 2; Starling, 2; House Sparrow, 77.5; Brewer's Blackbird, 1; Pine Grosbeak, 19; Hoary Redpoll, 2; Common Redpoll, 523; Snow Bunting, 1.-A. Binnie, Dr. R. M. Bremner, Murray Cox, Betty Gerrard, Dr. J. Gerrard, Jonathan Gerrard, George Gerrity, Ross Gerrity, J. B. Gollop, Michael Gollop, Mrs. V. Harper, Grace Hogg, Jim Hogg, Jean Mackenzie, H. McLennan, Michael Miller, A. Plumstead (Toronto), W. Plumstead, J. F. Roy (compiler), John Shadick, Lindy Lou Wedge, Terry Wedge, T. Wedge (Members and friends of the Saskatoon Natural History Society.)

SKULL CREEK, Sask. Dec. 27; 4 miles on foot and 5 by car in $2 \frac{1}{2}$ hours, plus observations in four farmyards; temp. $26^{\circ}$; wind light; clear; no snow. 15 species, 787 individuals. Rough-legged Hawk, 2; Pigeon Hawk, 1; Sparrow Hawk, 1; Sharp-tailed Grouse, 55; Ringnecked Pheasant, 5; Gray Partridge, 14; Great Horned Owl, 3; Downy Woodpecker, 3; Black-billed Magpie, 63; Black-capped Chickadee, 34; Bo- hemian Waxwing, 119; House Sparrow, 385; Pine Grosbeak, 3; Common Redpoll, 57; Snow Bunting, 42. (Add: Golden Eagle, 2, Dec. 23; Prairie Falcon, 1, Jan. 1; Short-eared Owl, 2, Dec. 24; Horned Lark, 2, Jan. 2; Northern Shrike, 1, Dec. 22; Redwinged Blackbird, 1, Dec. 20.)-Mrs. Lena Bennetto, Betty and Bob Mann, Mr. and Mrs. S. A. Mann (compilers), Henry Porman, Helen and Ray Schuler, Reid Shearwood, Peter and Dionna Swain, Harry Williams, Kenny Wright.

SPIRIT LAKE, Sask. Dec. 27; 9 party miles in 9 hours; temp. $20^{\circ}$; moderate north wind and light snow; 11 inches of snow on ground. 14 species, 301 individuals. Ruffed Grouse, 6; Sharp-tailed Grouse, 19; Great Horned Owl, 1; Hairy Woodpecker, 6; Downy Woodpecker, 7; Black-billed Magpie, 5; Black-capped Chickadee, 63; White-breasted Nuthatch, 1; Bohemian Waxwing, 2; House Sparrow, 41; Pine Grosbeak, 6; Common Redpoll, 18; Song Sparrow, 1; Snow Bunting, 125. (Add: Evening Grosbeak, Dec. 26.) - Bill Anaka, Joyce Gunn, June Popowich.

STRUAN, Sask. Dec. 24; 5 miles by car and 6 miles on foot; temp. $15^{\circ}$; wind $\mathrm{SE}$ at $10 \mathrm{mph}$; very little snow; clear. 9 species, 155 individuals. Ruffed Grouse, 2; Sharp-tailed Grouse, 4; Rock Dove, 3; Great Horned Owl, 1; Black-billed Magpie, 5; Black-capped Chickadee, 2; House Sparrow, 39; Common Redpoll, 94; Snow Bunting. 5. (Add: Gray Partridge, 6, Dec. 23; Bohemian Waxwing, 4, Dec. 26; Starling, 2, Dec. 27.)-William E. Jasper.

WOODROW, Sask. Dec. 26; on foot 18 miles along Wood River and Pinto Creek and return by car; temp. $26^{\circ}$; wind NNW 20 mph.; lightly overcast. 8 species, 449 individuals. Golden Eagle, 2; Sharp-tailed Grouse, 2; Ring-necked Pheasant, 55; Gray Partridge, 8; Owl (sp.?), 6; Blackbilled Magpie, 20; Black-capped Chickadee, 6; House Sparrow, 250. (Add: Snow Bunting, 75, Dec. 23.)Fred C. Parchman.

YORKTON, Sask. Dec. 26; 81/2 party miles in 5 hours on foot, 71 party miles in 10 hours by car; 15 observers in 4 parties; temp. $24^{\circ}$; wind $\mathrm{N}$ at $10 \mathrm{mph}$; 10 inches of snow. 10 species, 1498 individuals. Ruffed Grouse, 7; Gray Partridge, 16; Great 
Horned Owl, 2; Downy Woodpecker, 1; Black-billed Magpie, 8; Blackcapped Chickadee, 14; Bohemian Waxwing, 6; House Sparrow, 1156; Common Redpoll, 119; Snow Bunting, 169. (Add: Hairy Woodpecker, 1, Dec. 28.)-Gary Anweiler, Henry Chilman,
Jr., Tom Cursons, Archie Fraser, Art Gellert, Dr. and Mrs. Stuart Houston (compilers), Dr. C. J. Houston, Stanley Houston, Phil Pawluck, Elwood Sharpe, Jeff Smith, Donald Swaby, Frank Switzer, Guillain Switzer (Yorkton Natural History Society.)

\title{
Distraction Display By Western Meadowlark
}

\author{
By Sam Alberts, Brooks, Alberta
}

I was very interested in the article in the September, 1959, issue of the Blue Jay by Robert Nero "Distraction Display by Western Meadowlark." On the evening of July 27, 1959, I drove my truck up to the edge of a field that I was starting to irrigate. I noticed a meadowlark fly up and then come down to the ground and start doing the broken wing act as it went through the grass. My first thought was that it was a young one and that I had run over it, then I noticed it fly up on to a fence post so I knew it wasn't injured. It being so late in the summer I didn't think there would be a nest. However, when the same thing happened the next day I started looking for a nest, and found that if I had driven about two feet further I would have run over it. There were three young that looked about half-grown. I drove up to the same place about a dozen times on the 28th and 29th and each time the mother bird went through the same antics. At the time it seemed rather odd to see a meadowlark doing this broken wing act, but I did not know it was such an unusual thing or I would have watched it more closely and tried to get some pictures. We left for a few days holidays on the 30 th and when I came back on August 7 the nest was empty.

Note: Readers may be interested in similar report for the Westerm
Meadowlark by J. M. Linsdale (1938. Environmental responses of vertebrates in the Great Basin. Amer. Midland Nat., 19:1-206). On page 127 Linsdale states: "A brooding bird flew up just as it was almost stepped on. It landed 2 feet away and ran, fluttering along the ground for 50 feet, disappearing beneath a clump of buffalo berry." And Du Bois, quoted by A. C. Bent (1958. Life histories of North American blackbirds, orioles, tanagers, and Allies, U.S. Nat.' Mus. Bull. 211:36) describes nearly identical behaviour for the Bobolink: "The female jumps over the grass for a distance of three or four feet, then hobbles along in the grass; and, if I follow her, she repeats this-and continues to repeat until we are perhaps a hundred feet from the nest, then she flies for a short distance. This is the pattern of her ruse." "Bent points out that one reason for the difficulty of locating Bobolink nests is the female's habit of running through the grass for some distance from the nest before flushing (loc.cit: 35 ).

It seems apparent that in these two species this type of "distraction display" provides an effective means of escape from the nest (thus saving the nest), at least in some types of cover, as well as being a ruse to lure away predators. 